\title{
ON THE SPECTRUM OF ALMOST PERIODIC SOLUTIONS OF AN ABSTRACT DIFFERENTIAL EQUATION
}

\section{ARIBINDI SATYANARAYAN RAO AND WALTER HENGARTNER}

(Received 20 November 1972)

Communicated by J. P. O. Silberstein

\begin{abstract}
If a linear operator $A$ in a Banach space satisfies certain conditions, then the spectrum of any almost periodic solution of the differential equation $u^{\prime}=A u+f$ is shown to be identical with the spectrum of $f$, where $f$ is a Stepanov almost periodic function.
\end{abstract}

1

Suppose $X$ is a Banach space and $J$ is the interval $-\infty<t<\infty$. A continuous function $f: J \rightarrow X$ is said to be (Bochner or strongly) almost periodic if, given $\varepsilon>0$, there is a positive real number $\ell=\ell(\varepsilon)$ such that any interval of the real line of length $\ell$ contains at least one point $\tau$ for which

$$
\sup _{t \in J}\|f(t+\tau)-f(t)\| \leqq \varepsilon .
$$

For $1 \leqq p<\infty$, a function $f \in L_{\text {loc }}^{p}(J ; X)$ is said to be Stepanov almost periodic or $S^{p}$-almost periodic if, given $\varepsilon>0$, there exists a positive real number $\ell=\ell(\varepsilon)$ such that any interval of the real line of length $\ell$ contains at least one point $\tau$ for which

$$
\sup _{t \in J}\left[\int_{t}^{t+1}\|f(s+\tau)-f(s)\|^{p} d s\right]^{1 / p} \leqq \varepsilon .
$$

It is known that, for an $S^{p}$-almost periodic $X$-valued function $f(t)$ and a real number $\lambda$, the mean value

$$
m\left(e^{-i \lambda t} f(t)\right)=\lim _{T \rightarrow \infty} \frac{1}{T} \int_{0}^{T} e^{-i \lambda t} f(t) d t
$$

exists in $X$ and is different from the null element $\theta$ of $X$ for at most a countable 
set $\left\{\lambda_{n}\right\} n \geqq 1$, called the spectrum of $f(t)$ (see Theorem 9, page 79, Amerio-Prouse [1]). We denote by $\sigma(f(t))$ the spectrum of $f(t)$.

Our main result is as follows.

THEOREM 1. Suppose $A$ is a closed linear operator with domain $D(A)$ in a Banach space $X,(i \lambda-A)^{-1}$ exists for all real $\lambda$, and $f: J \rightarrow X$ is an $S^{p_{\text {-almost }}}$ periodic continuous function with $1 \leqq p<\infty$. If a continuously differentiable function $u: J \rightarrow D(A)$ is an almost periodic solution of the differential equation

$$
u^{\prime}(t)=A u(t)+f(t) \text { on } J,
$$

then $\sigma(u(t))=\sigma(f(t))$.

\section{Proof of Theorem 1}

We have

$$
\begin{aligned}
\frac{1}{T} \int_{0}^{T} e^{-i \lambda t} u^{\prime}(t) d t & =\frac{1}{T}\left[e^{-i \lambda t} u(t)\right]_{0}^{T}+\frac{i \lambda}{T} \int_{0}^{T} e^{-i \lambda t} u(t) d t \\
& \rightarrow i \lambda m\left(e^{-i \lambda t} u(t)\right) \text { as } T \rightarrow \infty .
\end{aligned}
$$

By (1.4) and (2.1), since $A$ is a closed linear operator,

$$
\begin{aligned}
\lim _{T \rightarrow \infty} \frac{1}{T} \int_{0}^{T} e^{-i \lambda t} A u(t) d t & =\lim _{T \rightarrow \infty} A\left(\frac{1}{T} \int_{0}^{T} e^{-i \lambda t} u(t) d t\right) \\
& =i \lambda m\left(e^{-i \lambda t} u(t)\right)-m\left(e^{-i \lambda t} f(t)\right) .
\end{aligned}
$$

So, again by the closedness of the operator $A$, since

$$
\lim _{T \rightarrow \infty} \frac{1}{T} \int_{0}^{T} e^{-i \lambda t} u(t) d t=m\left(e^{-i \lambda t} u(t)\right)
$$

exists in $X$, we have

Hence

$$
\left\{\begin{array}{l}
m\left(e^{-i \lambda t} u(t)\right) \in D(A) \text { and } \\
A m\left(e^{-i \lambda t} u(t)\right)=i \lambda m\left(e^{-i \lambda t} u(t)\right)-m\left(e^{-i \lambda t} f(t)\right) .
\end{array}\right.
$$

$$
(i \lambda-A) m\left(e^{-i \lambda t} u(t)\right)=m\left(e^{-i \lambda t} f(t)\right) .
$$

By our hypothesis, the operator $(i \lambda-A)$ is $1-1$ for all real $\lambda$. So it follows that

$$
m\left(e^{-i \lambda t} u(t)\right)=\theta \text { if and only if } m\left(e^{-i \lambda t} f(t)\right)=\theta,
$$

which completes the proof of the theorem.

\section{Now we establish the following result}

THEOREM 2. Let $B$ be a bounded linear operator in a Banach space $X$ such that 


$$
\left\|e^{\boldsymbol{t}}\right\| \leqq e^{a t} \text { for some } a<0 \text { and all } t \geqq 0 \text {. }
$$

Further, let $g: J \rightarrow X$ be an $S^{p}$-almost periodic continuous function with $1 \leqq p$ $<\infty$. Then the differential equation

$$
v^{\prime}(t)=B v(t)+g(t) \text { on } J
$$

has a unique almost periodic solution $v(t)$. Moreover, we have $\sigma(v(t))=\sigma(g(t))$.

Proof. Since the $S^{p}$-almost periodicity of $g$ implies the $S^{1}$-almost periodicity of $g$, it is sufficient to consider the case $p=1$. The proof of the uniqueness and existence of an almost periodic solution $v(t)$ of the differential equation (3.2) given by Zaidman [3] for $p=2$ in a Hilbert space goes through for $p=1$ in any Banach space, with some minor modifications and by replacing the Cauchy-Schwarz inequality (wherever it occurs) by the corresponding Hölder's inequality for $p=1$ and $q=\infty$.

Further, by (3.1), we have

$$
\lim _{t \rightarrow \infty} \frac{\log \left\|e^{t B}\right\|}{t} \leqq a<0 .
$$

Therefore, by Theorem 11, p. 622, Dunford-Schwartz [2], the whole imaginary axis $\{i \lambda\}_{\lambda \in J}$ is contained in the resolvent set $\rho(B)$ of $B$. Consequently, by Theorem $1, \sigma(v(t))=\sigma(g(t))$.

The first author takes this opportunity to thank Professor S. Vasilach for the financial support from his Government of Quebec grant during the preparation of this paper.

\section{References}

[1] L. Amerio, and G. Prouse, Almost periodic functions and functional equations, (Van Nostrand Reinhold Company (1971)).

[2] N. Dunford, and J. T. Schwartz, Linear operators, part I, (Interscience Publishers, Inc., New York (1958)).

[3] S. Zaidman, 'An existence result for Stepanoff almost periodic differential equations', Canad. Math. Bull. 14 (1971), 551-554.

Department of Mathematics

Université Laval

Québec City, P. Québec

Canada 